$W$ is $n$-parallelisable. Now by the main theorem of [2], $\partial W$ bounds a contractible manifold, and so represents the zero element of $\Gamma_{2 n}$.

\title{
REFERENCES
}

1. C. T. C. Wall, Classification of ( $n-1)$-connected $2 n$-manifolds, Ann. of Math. 75 (1962), 163-189.

2. - Killing the middle homotopy group of odd dimensional manifolds, Trans. Amer. Math. Soc. 103 (1962), 421-433.

Trinity College, Cambridge

\section{THE COEFFICIENTS IN THE EXPANSION OF CERTAIN PRODUCTS}

L. CARLITZ 1

1. The identities

(1)

$$
\begin{aligned}
\prod_{n=0}^{\infty}\left(1-p^{n} x\right)^{-1} & =\sum_{n=0}^{\infty} \frac{x^{n}}{(1-p)\left(1-p^{2}\right) \cdots\left(1-p^{n}\right)}, \\
\prod_{n=0}^{\infty}\left(1-p^{n} x\right) & =\sum_{n=0}^{\infty} \frac{(-1)^{n} p^{n(n-1) / 2} x^{n}}{(1-p)\left(1-p^{2}\right) \cdots\left(1-p^{n}\right)},
\end{aligned}
$$

where $|p|<1$, are well known. The more general products

$$
\prod_{m, n=0}^{\infty}\left(1-p^{m} q^{n} x\right)^{-1}, \quad \prod_{m, n=0}^{\infty}\left(1-p^{m} q^{n} x\right) \quad(|p|<1,|q|<1)
$$

have been discussed in $[1 ; 2]$.

In the present note we consider the products

(3) $\prod_{n=0}^{\infty}\left(1-p^{n} x-q^{n} y\right)^{-1}, \prod_{n=0}^{\infty}\left(1-p^{n} x-p^{n} y\right)(|p|<1,|q|<1)$.

Put

$$
F(x, y)=\prod_{n=0}^{\infty}\left(1-p^{n} x-q^{n} y\right)^{-1}=\sum_{r, 8=0}^{\infty} A_{r s} x^{r} y^{s},
$$

where $A_{r s}=A_{r s}(p, q)$ is independent of $x$ and $y$. It follows from (4) that

Presented to the Society, November 10,1961; received by the editors November 7, 1961.

${ }^{1}$ Supported in part by National Science'Foundation grant G-16485. 


$$
(1-x-y) F(x, y)=F(p x, q y)
$$

so that

$$
\left(1-p^{r} q^{s}\right) A_{r s}=A_{r-1,8}+A_{r, 8-1} \quad(r+s>0) .
$$

Making use of (5) we get for the first few values of $A_{r s}$ :

$$
\begin{aligned}
& A_{00}=1, \quad A_{10}=\frac{1}{1-p}, \quad A_{01}=\frac{1}{1-q}, \\
& A_{20}=\frac{1}{(1-p)\left(1-p^{2}\right)}, \quad A_{02}=\frac{1}{(1-q)\left(1-q^{2}\right)}, \\
& A_{11}=\frac{1}{(1-p)(1-p q)}+\frac{1}{(1-q)(1-p q)} .
\end{aligned}
$$

It is evident from (1) that

(6)

$$
\begin{aligned}
& A_{r 0}=\frac{1}{(1-p)\left(1-p^{2}\right) \cdots\left(1-p^{r}\right)}, \\
& A_{0 r}=\frac{1}{(1-q)\left(1-q^{2}\right) \cdots\left(1-q^{r}\right)} .
\end{aligned}
$$

Also it is clear from (3) that

$$
A_{r s}(p, q)=A_{s r}(q, p) \text {. }
$$

In (5) take $s=1$, so that

$$
\left(1-p^{r} q\right) A_{r 1}=A_{r-1,1}+A_{r 0} .
$$

Making use of (6) and (8) we find that

(9) $A_{r 1}=\sum_{j=0}^{r} \frac{1}{(1-p) \cdots\left(1-p^{j}\right)\left(1-p^{j} q\right) \cdots\left(1-p^{r} q\right)}$.

We next take $s=2$ in (5) and combine with (9) to get

(10) $A_{r 2}=\sum_{0 \leqq j \leqq k \leqq r} \frac{1}{(1-p) \cdots\left(1-p^{j}\right)\left(1-p^{j} q\right) \cdots\left(1-p^{k} q\right)\left(1-p^{k} q^{2}\right) \cdots\left(1-p^{r} q^{2}\right)}$.

It is now not difficult to state the general result, namely

(11) $A_{r s}=\sum \frac{1}{(1-p) \cdots\left(1-p^{i_{1}}\right)\left(1-p^{i_{1}} q\right) \cdots\left(1-p^{i_{2}} q\right) \cdots\left(1-p^{i^{s}} q^{8}\right) \cdots\left(1-p^{r} q^{8}\right)}$, where the summation is over all $j_{1}, j_{2}, \cdots, j_{\text {s }}$ such that

$$
0 \leqq j_{1} \leqq j_{2} \leqq \cdots \leqq j_{s} \leqq r .
$$


The proof of (11) is by induction on $s$ and will be omitted.

As a partial verification of (11) we note that since the number of solutions of (12) is equal to

$$
\left(\begin{array}{c}
r+s \\
r
\end{array}\right)=\frac{(r+s) !}{r ! s !}
$$

it follows that when $p=q$, (11) reduces to

$$
A_{r s}(p, q)=\left(\begin{array}{c}
r+s \\
r
\end{array}\right) \frac{1}{(1-p) \cdots\left(1-p^{r+s}\right)}
$$

which agrees with (1).

2. Turning next to the second product in (3) we put

$$
G(x, y)=\prod_{n=1}^{\infty}\left(1-p^{n} x-q^{n} y\right)=\sum_{r, s=0}^{\infty} B_{r s} x^{r} y^{s} .
$$

It follows from (13) that

$$
(1-p x-q y) G(p x, q y)=G(x, y),
$$

so that

$$
\left(1-p^{r} q^{s}\right) B_{r s}=-p^{r} q^{s}\left(B_{r-1,8}+B_{r, 8-1}\right) .
$$

Also comparing (13) with (2) we have

$$
B_{r 0}=\frac{(-1)^{r} p^{r(r+1) / 2}}{(1-p) \cdots\left(1-p^{r}\right)} .
$$

Now put

$$
B_{r s}^{*}=B_{r s}^{*}(p, q)=B_{r s}\left(\frac{1}{p}, \frac{1}{q}\right) .
$$

Then (14) becomes

$$
\left(1-p^{r} q^{8}\right) B_{r s}^{*}=B_{r-1, s}^{*}+B_{r, 8-1}^{*} .
$$

Since by (15)

$$
B_{r 0}^{*}=\frac{1}{(1-p) \cdots\left(1-p^{r}\right)}=A_{r 0},
$$

comparison of (16) with (5) yields

$$
B_{r s}^{*}=A_{r s} .
$$


Therefore we have

$$
B_{r s}(p, q)=A_{r s}\left(\frac{1}{p}, \frac{1}{q}\right)
$$

and $B_{r s}$ is determined explicitly by means of (11).

If we let $A_{r s}\left(j_{1}, \cdots, j_{s}\right)$ denote the summand in the right member of (11) and $A_{r s}^{*}\left(j_{1}, \cdots, j_{s}\right)$ the corresponding function with $p, q$ replaced by $p^{-1}, q^{-1}$, respectively, it follows that

$$
\begin{aligned}
A_{r s}^{*}\left(j_{1}, \cdots, j_{s}\right)= & (-1)^{r+s} p^{r(r+1) / 2+j_{1}+\cdots+j_{s}} \\
& q^{s(s+1) / 2+r s-j_{1}-\cdots-j_{s}} \cdot A_{r s}\left(j_{1}, \cdots, j_{8}\right) .
\end{aligned}
$$

Note that when $p=q$ the sum of the exponents on $p$ and $q$ is equal to

$$
\frac{1}{2} r(r+1)+\frac{1}{2} s(s+1)+r s=\frac{1}{2}(r+s)(r+s+1),
$$

which is correct.

In terms of $A_{r s}\left(j_{1}, \cdots, j_{s}\right)$ and $A_{r s}^{*}\left(j_{1}, \cdots, j_{s}\right)$ we have

$$
\begin{aligned}
& A_{r s}=\sum A_{r s}\left(j_{1}, \cdots, j_{s}\right), \\
& B_{r s}=\sum A_{r s}^{*}\left(j_{1}, \cdots, j_{s}\right),
\end{aligned}
$$

where in each case the summation is over all $j_{1}, \cdots, j_{s}$ satisfying (12).

From the definition of $A_{r s}\left(j_{1}, \cdots, j_{s}\right)$ we have

$$
A_{r s}\left(j_{1}, \cdots, j_{s}\right)=\frac{A_{j_{s, s-1}}\left(j_{1}, \cdots, j_{s-1}\right)}{\left(1-p^{j_{s}} q^{s}\right) \cdots\left(1-p^{r} q^{s}\right)} ;
$$

therefore (20) yields

$$
A_{r s}=\sum_{j=0}^{r} \frac{A_{j, 8-1}}{\left(1-p^{j} q^{s}\right) \cdots\left(1-p^{r} q^{s}\right)},
$$

which can also be obtained from (5).

We remark that the pair of formulas

$$
\begin{aligned}
& (r+1) A_{r+1, s}=\sum_{j=0}^{r} \sum_{k=0}^{s}\left(\begin{array}{c}
j+k \\
j
\end{array}\right) \frac{A_{r-j, s-k}}{1-p^{j+1} q^{k}}, \\
& (s+1) A_{r, 8+1}=\sum_{j=0}^{r} \sum_{k=0}^{s}\left(\begin{array}{c}
j+k \\
j
\end{array}\right) \frac{A_{r-j, s-k}}{1-p^{j} q^{k+1}}
\end{aligned}
$$

can be proved by logarithmic differentiation of (4).

3. We shall now determine the coefficients in the expansion 


$$
\prod_{n=0}^{\infty}\left(1-p_{1} x_{1}-p_{2} y-p_{3} z\right)^{-1}=\sum_{r, s, t=0} A_{r s t} x^{r} y^{s} z^{t}
$$

A triple $T$ is an ordered set of three non-negative integers $i, j, k$. Each of the triples $(i-1, j, k),(i, j-1, k),(i, j, k-1)$ precedes $i, j, k$; notation $T_{1} \prec T$. A chain $C$ is a set of triples:

$$
T_{1} \prec T_{2} \prec \cdots<T_{k},
$$

where $T_{1}=(1,0,0),(0,1,0)$ or $(0,0,1) ; T_{k}$ is the last element of $C$. Corresponding to the triple $i, j, k$ is the factor $1-p_{1}^{i} p_{2}^{j} p_{3}^{k}$; we put

$$
\pi(C)=\prod_{T \in C}\left(1-p_{1}^{i} p_{2}^{j} p_{3}^{k}\right)
$$

where $1-p_{1}^{i} p_{2}^{j} p_{3}^{k}$ corresponds to the triple $T=(i, j, k)$.

We shall show that

$$
A_{r s t}=\sum_{C} \frac{1}{\pi(C)},
$$

where the summation is over all chains with last element $(r, s, t)$.

In the first place it is clear from (25) that

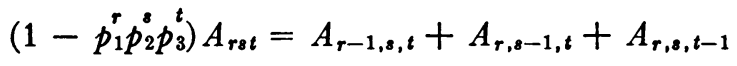

and

$$
A_{r s 0}=A_{r s}\left(p_{1}, p_{2}\right), \quad A_{r-t}=A_{r t}\left(p_{1}, p_{3}\right), \quad A_{0 s t}=A_{s t}\left(p_{2}, p_{3}\right),
$$

where $A_{r s}\left(p_{1}, p_{2}\right)$ is defined by (4). Moreover $A_{r s t}$ is uniquely determined by means of (28) and (29).

Now if $A_{r s t}$ is defined by (27) it follows from (11) and (27) that (28) is satisfied. To show that (28) is also satisfied we remark that if $C$ is a chain with last element $(r, s, t)$, then deleting this element we are left with a chain whose last element is $(r-1, s, t),(r, s-1, t)$ or $(r, s, t-1)$ and conversely. In view of (26), (28) follows immediately.

If we put

$$
\prod_{n=1}^{\infty}\left(1-p_{1} x-p_{2} y-p_{3} z\right)=\sum_{r, s, t=0}^{\infty} B_{r s t} x^{r} y^{s} z^{t}
$$

then, exactly as in the proof of (18), we have

$$
B_{r s t}=A_{r s t}\left(\frac{1}{p_{1}}, \frac{1}{p_{2}}, \frac{1}{p_{8}}\right) .
$$

It is clear from (26), (27) and (31) how the coefficients in the expansion of 
1962] THE COEFFICIENTS IN THE EXPANSION OF CERTAIN PRODUCTS 949

$$
\prod_{n=0}^{\infty}\left(1-p_{1} x_{1}-\cdots-p_{k} x_{k}\right)^{-1}
$$

and

$$
\prod_{n=1}^{\infty}\left(1-p_{1} x_{1}+\cdots+p_{k} x_{k}\right)
$$

can be determined for all $k \geqq 1$.

Added in proof. Professor B. M. Bennett has kindly informed the writer that he has obtained a formula equivalent to (11) above in his paper: On a rank-order test for the equality of probabilities in multinomial trials. Also it is evident from his paper that the coefficients $A_{r s}(p, q)$ are of some statistical interest.

\section{REFERENCES}

1. L. Carlitz, The expansion of certain products, Proc. Amer. Math. Soc. 7 (1956), 558-564.

2. E. M. Wright, Partitions of multi-partite numbers, Proc. Amer. Math. Soc. 7 (1956), 880-890.

DURE UNIVERSITY 